\title{
Strategies to augment growth-hormone secretion in obesity
}

\author{
Steven Grinspoon
}

Obesity is estimated to affect 300 million people worldwide. Furthermore, abdominal adiposity-measured by waist circumferenceis independently associated with mortality. This association remains significant, even after accounting for total weight, which suggests independent effects of central fat accumulation. A reduction in growth-hormone $(\mathrm{GH})$ secretion has been reported in patients with obesity, and is characterized by reduced pulse height and width, but preserved pulse frequency. Around a third of individuals with a BMI $>30 \mathrm{~kg} / \mathrm{m}^{2}$ fail a standard arginine plus GH-releasing hormone $(\mathrm{GHRH})$ stimulation test. Excess visceral adiposity is associated with reduced $\mathrm{GH}$ secretion in such individuals: the peak, stimulated $\mathrm{GH}$ concentration is reduced by $1 \mu \mathrm{g} / \mathrm{l}$ for each $1 \mathrm{~cm}$ increase in waist circumference. The mechanism by which visceral fat is associated with reduced $\mathrm{GH}$ secretion is not clear, but reduction of excess lipolytic rates that result from increased adiposity results in increased $\mathrm{GH}$ secretion. Increased visceral fat could contribute to reduced $\mathrm{GH}$ secretion, which in turn might lead to further increases in visceral fat, which thereby promotes a vicious cycle.

Reduced $\mathrm{GH}$ secretion is associated with increased mortality and cardiovascular disease in patients with pituitary-tumor-associated $\mathrm{GH}$ deficiency. In patients with obesity, reduced $\mathrm{GH}$ secretion correlates with dyslipidemia, increased inflammation and increased carotid intima-media thickness. These data suggest that reduced $\mathrm{GH}$ secretion might mediate some of the excess cardiovascular risk associated with obesity

Should endogenous GH secretion be augmented in patients with abdominal obesity? Administration of $\mathrm{GH}$ can markedly reduce visceral fat and improve lipid profiles. By contrast, mixed effects are observed on glucose homeostasis. Glycemia initially worsens upon initiation of GH therapy; however, improvements can occur over time, as the insulin-antagonistic effects of $\mathrm{GH}$ are outweighed by beneficial
Development of augmentation strategies that improve pulsatile $\mathrm{GH}$ secretion might uniquely target visceral fat...

$S$ Grinspoon is a Professor of Medicine, Director of the Program in Nutritional Metabolism and Clinical Director of the Neuroendocrine Clinical Center, Massachusetts General Hospital and Harvard Medical School, Boston, MA, USA.

\section{Competing interests}

The author declared associations with the following companies: Elixir Pharmaceuticals,

Serono and

Theratechnologies. See the article online for full details of the relationships.

www.nature.com/clinicalpractice doi:10.1038/ncpendmet1075 reductions in visceral fat. The initial worsening of glucose concentration could relate to the nonphysiological mode of $\mathrm{GH}$ administration, with a single, large, nonpulsatile bolus given daily. Ironically, such dosing might actually decrease endogenous $\mathrm{GH}$ secretion between doses, particularly if the dose is supraphysiological.

An alternative approach is to use agents that increase pulsatile secretion of $\mathrm{GH}$ and so address a fundamental abnormality of obesity. Administration of GHRH can reduce visceral fat, improve lipid profiles and increase adiponectin levels in patients with HIV and acquired visceral adiposity. Interestingly, GHRH specifically reduced visceral, rather than subcutaneous, fat in patients with lipodystrophy. Administration of either $\mathrm{GH}$ or $\mathrm{GHRH}$ to patients with lipodystrophy caused identical physiological increases in insulin-like growth factor I (IGF-I) levels; however, visceral fat was most reduced in the patients who received GHRH. Moreover, $2 \mathrm{~h}$ blood-glucose levels increased in response to $\mathrm{GH}$, but not $\mathrm{GHRH}$, administration. Whether such differences reflect a physiologic effect of $\mathrm{GHRH}$ on endogenous pulsatile secretion of $\mathrm{GH}$ remains unclear. An additional advantage of $\mathrm{GHRH}$ is that feedback inhibition via IGF-I remains intact. In contrast to $\mathrm{GHRH}$, ghrelin and ghrelin-like peptides stimulate $\mathrm{GH}$ through the endogenous $\mathrm{GH}$-secretagogue receptor, with some crosstalk to the $\mathrm{GHRH}$ receptor. These agonists can potentially increase $\mathrm{GH}$ secretion; however, they are not specific to $\mathrm{GH}$, and could increase secretion of other pituitary hormones (e.g. cortisol). In addition, they are orexigenic.

Further investigation is necessary to determine whether $\mathrm{GHRH}$ and other $\mathrm{GH}$ secretagogues will prove useful to augment endogenous $\mathrm{GH}$ secretion, reduce visceral fat, and improve metabolic parameters in individuals with generalized obesity. Development of augmentation strategies that improve pulsatile $\mathrm{GH}$ secretion might uniquely target visceral fat, and so represent a novel approach to the treatment of obesity. 